\title{
AN EQUIVARIANT CONSTRUCTION
}

\author{
PEDRO L. Q. PERGHER \\ (Communicated by Frederick R. Cohen)
}

\begin{abstract}
In this paper we show how to construct the equivariant bordism class of a closed manifold with differentiable $\left(Z_{2}\right)^{k}$-action from its fixed data.
\end{abstract}

\section{INTRODUCTION}

In [1] Conner and Floyd proved that the equivariant bordism class of a closed manifold with involution $\left(M^{n}, T\right)$ is completely determined by the fixed data $(F, \eta)$, where $F$ is the fixed point set and $\eta$ is its normal bundle. In particular, $M$ is bordant to $R P(\eta \oplus 1)$.

In [3] Stong showed more generally that for $\left(Z_{2}\right)^{k}$-actions the equivariant bordism class is also determined by the fixed data, which consists in this case of the fixed point set and the subbundles of the normal bundle on which $\left(Z_{2}\right)^{k}$ has given representations. How to construct the bordism class of the original manifold in terms of that fixed data was described in [2].

The object of this note is to complete [2] by showing that the mentioned construction can actually be carried through equivariantly; more precisely, we will exhibit a manner to construct a $\left(Z_{2}\right)^{k}$-action on that construction recovering the bordism class of the original action.

\section{Preliminaries}

Throughout this paper, $\left(Z_{2}\right)^{k}$ will be considered as the group generated by $k$ commuting involutions $T_{1}, T_{2}, \ldots, T_{k}$. Let $\left(M^{n}, \varphi\right), \varphi=\left(T_{1}, T_{2}, \ldots, T_{k}\right)$, be a $\left(Z_{2}\right)^{k}$-action with $F$ as fixed point set (we shall use the notation $F=F_{\varphi}$ ). The normal bundle $\eta$ of $F$ in $M^{n}$ decomposes as a Whitney sum of subbundles on which $\left(Z_{2}\right)^{k}$ acts as one of the irreducible (nontrivial) real representations. To make a description of this decomposition one may use sequences $a=\left(a_{1}, a_{2}, \ldots, a_{k}\right)$ where each $a_{j}$ is either 0 or 1 . Let $\varepsilon_{a} \subset \eta$ be the subbundle on which $T_{j}$ acts as multiplication by $(-1)^{a_{j}}$; then

$$
\eta=\bigoplus_{a \neq(0)} \varepsilon_{a}
$$

Received by the editors October 17, 1991 and, in revised form, January 17, 1992.

1991 Mathematics Subject Classification. Primary 57R85, 57R90; Secondary 57S17.

This work was partially supported by CNPq. 
where $(0)=(0,0, \ldots, 0)$ (trivial sequence). In this way, choosing an order for $\{a ; a \neq(0)\}, F$ and the ordered set of the $2^{k}-1$ vector bundles $\varepsilon_{a}$ constitute the fixed data of the action on $M^{n}$.

\section{THE CONSTRUCTION}

First, for $0 \leq i, j \leq k$, denote by $\mu_{i}$ the normal bundle of $F_{T_{i}}$ in $M^{n}$ and let $T_{j}^{*}$ be the involution on $R P\left(\mu_{i} \oplus 1\right)$ induced by 1 in the fibers of the trivial line bundle 1 and by the bundle map on $\mu_{i}$ covering $T_{j}$ on $F_{T_{i}}$. On $R P\left(\mu_{i} \oplus 1\right)$ we can also consider the involution $A_{T_{i}}$ induced by $1 \times(-1)$.

Recall that the construction of [2] was obtained by constructing a sequence of closed manifolds with $\left(Z_{2}\right)^{i}$-action $\left(M_{i}^{n}, \varphi_{i}\right), 0 \leq i \leq k$, starting with $\left(M^{n}, \varphi\right)$, with $M_{i-1}^{n}$ bordant to $M_{i}^{n}$ and with the fixed data of $\left(M_{i-1}^{n}, \varphi_{i-1}\right)$ being explicitly constructible from the fixed data of $\left(M_{i}^{n}, \varphi_{i}\right)$, thus providing the desired construction $M_{0}^{n}$. Recall still that the sequence above was obtained inductively by giving the description for the construction of $\left(M_{k-1}^{n}, \varphi_{k-1}\right)$ from $\left(M_{k}^{n}, \varphi_{k}\right)=\left(M^{n}, \varphi\right)$; specifically,

$$
\left(M_{k-1}^{n}, \varphi_{k-1}\right)=\left(R P\left(\mu_{k} \oplus 1\right), T_{1}^{*}, \ldots, T_{k-1}^{*}\right) .
$$

Now consider the $\left(Z_{2}\right)^{k}$-action $\left(R P\left(\mu_{i} \oplus 1\right), \psi_{i}\right)$ where $\psi_{i}=\left(T_{1}^{*}, \ldots, T_{i-1}^{*}\right.$, $\left.A_{T_{i}}, T_{i+1}^{*}, \ldots, T_{k}^{*}\right)$. To obtain our construction it suffices to prove that $\left[M^{n}, \varphi\right]$ $=\left[R P\left(\mu_{i} \oplus 1\right), \psi_{i}\right]$ in $\mathscr{N}_{n}\left(\left(Z_{2}\right)^{k}\right)$; indeed, in this case

$$
\begin{aligned}
{\left[M^{n}, \varphi\right] } & =\left[M_{k-1}^{n}, T_{1}^{*}, \ldots, T_{k-1}^{*}, A_{T_{k}}\right] \\
& =\left[M_{k-2}^{n},\left(T_{1}^{*}\right)^{*}, \ldots,\left(T_{k-2}^{*}\right)^{*}, A_{T_{k-1}^{*}}, A_{T_{k}}^{*}\right] \\
& =\cdots=\left[M_{0}^{n}, \sigma_{1}, \ldots, \sigma_{k}\right],
\end{aligned}
$$

the $\sigma_{i}$ 's being suggested by the fact above. We now proceed to prove this fact. One may form $M \times[-1,1]$ with the $\left(Z_{2}\right)^{k}$-action given by $T_{1} \times 1, T_{2} \times$ $1, \ldots, T_{k} \times 1$. On $M \times[-1,1]$ we can also consider the involution $T_{i} \times(-1)$, whose fixed data is $\mu_{i} \oplus 1 \rightarrow F_{T_{i}} \times\{0\}$. Let $W$ be the manifold obtained by removing the interior of an invariant tubular neighborhood of $F_{T_{i}} \times\{0\}$ (which is identified with the disc bundle $\left.D\left(\mu_{i} \oplus 1\right)\right)$ and by dividing out the free action of $T_{i} \times(-1)$. One notes that the boundary $\partial W$ is $M^{n} \cup R P\left(\mu_{i} \oplus 1\right)$. The involutions $T_{1} \times 1, \ldots, T_{k} \times 1$ induce an action on $W$ whose restriction to $\partial W$ is $\left(M^{n}, \varphi\right) \cup\left(R P\left(\mu_{i} \oplus 1\right), \psi_{i}\right)$, thus giving an equivariant cobordism from $\left(M^{n}, \varphi\right)$ to $\left(R P\left(\mu_{i} \oplus 1\right), \psi_{i}\right)$. This completes the proof.

\section{ACKNOWLEDGMENT}

I am very grateful to the referee for suggestions that helped to simplify and to clarify considerably the original version.

\section{REFERENCES}

1. P. E. Conner and E. E. Floyd, Differentiable periodic maps, Springer-Verlag, Berlin, 1964.

2. P. L. Q. Pergher, Manifolds with $\left(Z_{2}\right)^{k}$-actions, Proc. Amer. Math. Soc. 106 (1989), 10911094.

3. R. E. Stong, Equivariant bordism and $\left(Z_{2}\right)^{k}$-actions, Duke Math. J. 37 (1970), 779-785.

Departamento de Matemática, Universidade Federal de São Carlos, Rodovia Washington Luiz, KM 235, CEP 13560, São Carlos, SP, Brazil 